Check for updates

Cite this: J. Mater. Chem. C, 2022, 10, 4757

Received 13th October 2021, Accepted 6th December 2021

DOI: $10.1039 / \mathrm{d} 1 \mathrm{tc} 04918 \mathrm{f}$

rsc.li/materials-c

\section{Fluorinated dibenzo[a,c]-phenazine-based green to red thermally activated delayed fluorescent OLED emitters $\uparrow$}

\author{
Gloria Hong, (D) $\ddagger^{a}$ Changfeng Si, (D) $\ddagger^{\mathrm{b}}$ Abhishek Kumar Gupta, (D) $\ddagger^{\mathrm{bc}}$ \\ Claudia Bizzarri, (D) ${ }^{a}$ Martin Nieger, (D) ${ }^{d}$ Ifor D. W. Samuel, (D) *c \\ Eli Zysman-Colman (D) *b and Stefan Bräse (iD *ae
}

\begin{abstract}
Purely organic thermally activated delayed fluorescence (TADF) emitting materials for organic lightemitting diodes (OLEDs) enable a facile method to modulate the emission color through judicious choice of donor and acceptor units. Amongst purely organic TADF emitters, the development of TADF molecules that emit at longer wavelengths and produce high-efficiency devices that show low efficiency roll-off remains a challenge. We report a modular synthesis route that delivers three structurally related fluorinated dibenzo[a,c]-phenazine-based TADF molecules, each bearing two donor moieties with different electron-donating strengths, namely 3,6-bis(3,6-di-tert-butyl-9H-carbazol-9-yl)-10-fluorodibenzo[a,c]phenazine (2DTCz-BP-F), 3,6-bis(9,9-dimethylacridin-10(9H)-yl)-10-fluorodibenzo[a,c]phenazine (2DMAC-BP-F) and 10,10'-(10-fluorodibenzo[a,c]phenazine-3,6-diyl)bis(10H-phenoxazine) (2PXZ-BP-F). They exhibit donor strength-controlled color-tuning over a wide color range from green to deep-red with photoluminescence maxima, $\lambda_{\mathrm{PL}}$, of $505 \mathrm{~nm}, 589 \mathrm{~nm}$, and $674 \mathrm{~nm}$ in toluene solution. OLED devices using these TADF materials showed excellent to moderate performance with an $\mathrm{EQE}_{\max }$ of $21.8 \%$ in the case of 2DMAC-BP-F, $12.4 \%$ for 2 PXZ-BP-F and 2.1\% with 2DTCZ-BP-F, and associated electroluminescence (EL) emission maxima, $\lambda_{\mathrm{EL}}$, of $585 \mathrm{~nm}, 605 \mathrm{~nm}$ and $518 \mathrm{~nm}$ in an mCBP host, respectively.
\end{abstract}

\section{Introduction}

Among the emitting materials for use in organic light-emitting diodes (OLEDs), purely organic thermally activated delayed fluorescence (TADF) emitters have drawn intense interest in

\footnotetext{
${ }^{a}$ Institute of Organic Chemistry, Karlsruhe Institute of Technology (KIT), Fritz-Haber-Weg 6, 76131 Karlsruhe, Germany. E-mail: stefan.braese@kit.edu

${ }^{b}$ Organic Semiconductor Centre, EaStCHEM School of Chemistry,

University of St Andrews, St Andrews, Fife, KY16 9ST, UK.

E-mail: eli.zysman-colman@st-andrews.ac.uk; Fax: +44 (0)1334 463808; Tel: +44 (0)1334 463826

${ }^{c}$ Organic Semiconductor Centre, SUPA School of Physics and Astronomy, University of St Andrews, St Andrews, Fife, KY16 9SS, UK. E-mail: idws@st-andrews.ac.uk; Fax: +44 (0)1334 463104; Tel: +44 (0)1334 463826

${ }^{d}$ Department of Chemistry, University of Helsinki, P. O. Box 55, ooo14, Finland

${ }^{e}$ Institute of Biological and Chemical Systems (IBCS-FMS), Karlsruhe Institute of Technology (KIT), Hermann-von-Helmholtz-Platz 1,

76344 Eggenstein-Leopoldshafen, Germany

$\dagger$ Electronic supplementary information (ESI) available: General information, experimental procedures, compound characterization. CCDC 2086119 (2DMACBP-F). For ESI and crystallographic data in CIF or other electronic format see DOI: 10.1039/d1tc04918f

\$ Authors contributed equally to this work.
}

recent years as they enable devices to reach a theoretical internal quantum efficiency (IQE) of $100 \%$. This is possible through efficient harvesting of both singlet and triplet excitons to produce light, the latter of which are converted to the former via reverse intersystem crossing (RISC). Organic TADF emitters do not contain scarce, noble metals that are extracted through environmentally damaging mining operations. Swift progress has been reported in the development of purely organic TADF emitters and now there are numerous examples of TADF OLEDs showing comparable efficiencies to phosphorescent devices. ${ }^{1,2}$

RISC at ambient temperatures occurs in organic compounds that possess a small energy gap, $\Delta E_{\mathrm{ST}}$, between the lowest-lying singlet state $S_{1}$ and triplet state $T_{1}$, and show non-zero spinorbit coupling (SOC). ${ }^{3}$ For this scenario to occur, there must be a spatial separation of the electron-donating unit accommodating the highest-occupied molecular orbital (HOMO) and the electron-accepting unit hosting the lowest-unoccupied molecular orbital (LUMO). ${ }^{4}$ The implementation of this donor-acceptor molecular design produces a strong charge-transfer (CT) character of the $S_{1}$ state. $^{3}$

The design of TADF materials that emit at longer wavelengths poses some unique challenges for maintaining a high 
photoluminescence quantum yield ( $\left.\Phi_{\mathrm{PL}}\right)$. The $\Phi_{\mathrm{PL}}$ is dependent on the rate constant of radiative decay processes such as fluorescence, but also nonradiative decay processes such as internal conversion (IC) and intersystem crossing (ISC). ${ }^{5}$ In large, aromatic molecules, where the electronic relaxation lies within the rule of a weak coupling limit as reported by Englman and Jortner, ${ }^{6}$ the rate constant of the nonradiative decay, $k_{\mathrm{nr}}$, is inversely proportional to the exponential of the optical energy gap $\Delta E_{\text {opt }}$. In contrast, the rate constant of the radiative decay, $k_{\mathrm{r}}$, is proportional to the cube of $\Delta E_{\text {opt }} \cdot{ }^{5,7,8}$ As the energy of the emissive excited state decreases, the influence of nonradiative decay increases exponentially because the vibronic coupling between the excited state and ground state is facilitated. The challenge of reducing losses due to vibrational quenching and other nonradiative decay pathways in TADF molecules emitting at longer wavelengths can be partially addressed by introducing rigidity into the molecular structure of the donor and acceptor units. Common acceptors for purely organic TADF emitters are aromatic ketones such as anthraquinones, naphthalimides, or heteroaromatic systems like quinoxaline and dibenzo[ $a, c]$ phenazine (BP). ${ }^{9}$ These acceptors show deep LUMO levels of $-3.4 \mathrm{eV},{ }^{10}-2.99 \mathrm{eV},{ }^{11}-2.81 \mathrm{eV},{ }^{12}$ and $-2.90 \mathrm{eV},{ }^{13}$ respectively, that contribute to stabilizing the $S_{1}$ state and are therefore beneficial for use in the design of TADF emitters targeting longer wavelength regions.

Zhao and co-workers first reported TADF compounds bearing the $\mathrm{BP}$ acceptor, which exhibits a rigid, large $\pi$-conjugated system. ${ }^{14}$ These compounds contain one to three donor moieties in the donor-acceptor or poly(donor)-acceptor strategy, which are commonly applied for TADF molecule design. The greater number of 9,9-dimethyl-9,10-dihydroacridine (DMAC) donors was expected to strengthen the intramolecular charge transfer (ICT) and lead to color-tuning from green to orange-red emission with electroluminescence maxima, $\lambda_{\mathrm{EL}}$, of $560 \mathrm{~nm}$, $576 \mathrm{~nm}$, and $606 \mathrm{~nm}$ for devices featuring 1DMAC-BP, 2DMAC-BP, and 3DMAC-BP, respectively (Fig. 1). A maximum external quantum efficiency $\left(\mathrm{EQE}_{\max }\right)$ of $22.0 \%$ was observed for the OLED device with 3DMAC-BP doped in $\mathrm{mCBP}(18 \mathrm{wt} \%$ ) at $606 \mathrm{~nm}$.<smiles>[R]c1ccc2nc3c4ccc([R])cc4c4ccc(N5c6ccccc6C([Y16])c6ccccc65)cc4c3cc2c1</smiles>

1DMAC-BP: $\mathrm{R}=\mathrm{R}^{\prime}=\mathrm{H}$ 2DMAC-BP: $R=D M A C, R^{\prime}=H$ 3DMAC-BP: $R=R^{\prime}=$ DMAC

$\lambda_{\mathrm{EL}}=560 \mathrm{~nm}, 576 \mathrm{~nm}, 606 \mathrm{~nm}$ $\lambda_{\mathrm{PL}}=549 \mathrm{~nm}, 565 \mathrm{~nm}, 590 \mathrm{~nm}$ $\Phi_{\mathrm{PL}}=42 \%, 84 \%, 89 \%$ $\Delta \mathrm{E}_{\mathrm{ST}}=0.22 \mathrm{eV}, 0.21 \mathrm{eV}, 0.05 \mathrm{eV}$ $\tau_{\mathrm{d}}=12.6 \mu \mathrm{s}, 7.5 \mu \mathrm{s}, 2.9 \mu \mathrm{S}$ $E Q E_{\max }=10.1 \%, 11.8 \%, 22.0 \%$ ACS Appl. Mater. Interfaces 2019, 11, 26144-26151.

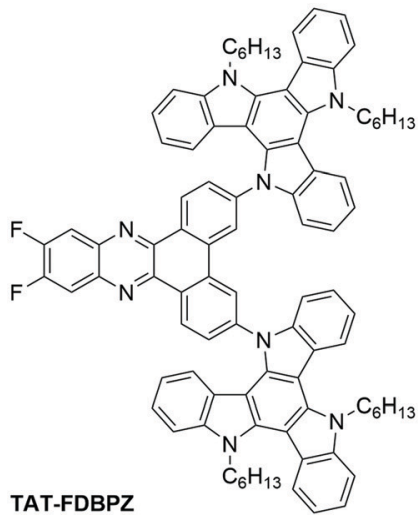

$$
\begin{aligned}
& \lambda_{\mathrm{EL}}=611 \mathrm{~nm} \\
& \lambda_{\mathrm{PL}}=593 \mathrm{~nm} \\
& \Phi_{\mathrm{PL}}=62 \% \\
& \Delta \mathrm{E}_{\mathrm{ST}}=0.10 \mathrm{eV} \\
& \tau_{\mathrm{d}}=1.51 \mu \mathrm{S} \\
& \mathrm{EQE}_{\max }=9.2 \%
\end{aligned}
$$$$
\text { ACS Appl. Mater. Interfaces 2020, 12, 30652-30658. }
$$<smiles></smiles>

1PXZ-BP: $R=R^{\prime}=H$ 2PXZ-BP: $R=P X Z, R^{\prime}=H$ 3PXZ-BP: $R=R^{\prime}=P X Z$

$\lambda_{\mathrm{EL}}=590 \mathrm{~nm}, 606 \mathrm{~nm}, 634 \mathrm{~nm}$ $\lambda_{\mathrm{PL}}=578 \mathrm{~nm}, 596 \mathrm{~nm}, 630 \mathrm{~nm}$ $\Phi_{\mathrm{PL}}=73 \%, 63 \%, 22 \%$ $\Delta \mathrm{E}_{\mathrm{ST}}=0.25 \mathrm{eV}, 0.10 \mathrm{eV}, 0.03 \mathrm{eV}$ $\tau_{\mathrm{d}}=4.8 \mu \mathrm{s}, 4.3 \mu \mathrm{s}, 2.0 \mu \mathrm{s}$ $\mathrm{EQE}_{\max }=26.3 \%, 19.2 \%, 7.1 \%$ Adv. Electron. Mater. 2020, 6, 1900843.

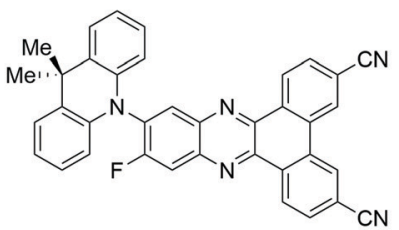

FBPCNAC

$$
\begin{aligned}
& \lambda_{\mathrm{EL}}=597 \mathrm{~nm} \\
& \lambda_{\mathrm{PL}}=607 \mathrm{~nm} \\
& \Phi_{\mathrm{PL}}=79 \% \\
& \Delta \mathrm{E}_{\mathrm{ST}}=0.047 \mathrm{eV} \\
& \tau_{\mathrm{d}}=11.1 \mu \mathrm{S} \\
& \mathrm{EQE}_{\max }=23.8 \% \\
& \text { ACS Appl. Mater. Interfaces 2020, 12, 16, 18730-18738. }
\end{aligned}
$$

Fig. 1 BP-based TADF emitters; DMAC: 9,9-dimethyl-9,10-dihydroacridine, PXZ: 10H-phenoxazine, DTCz: 3,6-di-tert-butylcarbazole. 
By employing the stronger donor $10 H$-phenoxazine (PXZ), the $\lambda_{\mathrm{EL}}$ for the devices with 1PXZ-BP, 2PXZ-BP, and 3PXZ-BP were red-shifted to $590 \mathrm{~nm}, 606 \mathrm{~nm}$, and $634 \mathrm{~nm}$, respectively. The most efficient device with 1PXZ-BP as the emitter showed an $\mathrm{EQE}_{\max }$ of $26.3 \%$ (7 wt\% doped in CBP). ${ }^{13}$ Both Lee and coworkers as well as Wang and coworkers, have reported fluorosubstituted BP acceptors intending to strengthen the acceptor with the presence of the strongly inductively electron-withdrawing fluorine substituent. ${ }^{15,16}$ Lee and coworkers reported the use of a fluorine substituent at the acceptor moiety in the ortho-position (FBPCNAc, Fig. 1) to the donor moiety. FBPCNAc is brightly luminescent in $1 \mathrm{wt} \%$ doped polystyrene film with $\lambda_{\mathrm{PL}}=607 \mathrm{~nm}$, a $\Phi_{\mathrm{PL}}$ of $79 \%$ and a delayed lifetime of $\tau_{\mathrm{d}}=11.1 \mu \mathrm{s}$. In the electroluminescent (EL) device, it showed an emission maximum of $\lambda_{\mathrm{EL}}=597 \mathrm{~nm}$, an $\mathrm{EQE}_{\max }$ of $23.8 \%$ and low efficiency roll-off. Wang and coworkers on the other hand, attached two fluorine substituents in 11- and 12-position to the $\mathrm{BP}$ acceptor on the opposite side of the donor moieties in 3- and 6-position (TAT-FDBPZ, Fig. 1) and observed that the introduction of the fluorine substituents led to a stronger ICT state and a red-shifted emission. The emission of the $20 \mathrm{wt} \%$ doped CBP films of the fluorinated TAT-FDBPZ is bathochromically shifted from $\lambda_{\mathrm{PL}}=593 \mathrm{~nm}$ by $17 \mathrm{~nm}$ in comparison to its nonfluorinated analogue $\left(\lambda_{\mathrm{PL}}=576 \mathrm{~nm}\right)$. However, this came at the cost of a slightly decreased $\Phi_{\mathrm{PL}}$ from $76 \%$ to $62 \%$. The $\tau_{\mathrm{d}}$ of TAT-FDBPZ is $1.51 \mu \mathrm{s}$, which is shorter than for the nonfluorinated analog where $\tau_{\mathrm{d}}=2.30 \mu \mathrm{s}$. The device based on TAT-FDBPZ showed $\lambda_{\mathrm{EL}}$ of $611 \mathrm{~nm}$ and an $\mathrm{EQE}_{\max }$ of $9.2 \%$.

Building on these findings, we aimed to reduce the molecular weight of the emitter in the context of vacuum-deposited device fabrication. We investigated a compromise of the poly(donor)-acceptor strategy by installing two donor units and an electron-withdrawing fluorine substituent to strengthen the dibenzo[a,c]phenazine acceptor. We designed three new TADF emitters, shown in Fig. 1, based on 10-fluorodibenzo$[a, c]$ phenazine (BP-F), each incorporating two donor moieties, namely 3,6-bis(3,6-di-tert-butyl-9H-carbazol-9-yl)-10-fluorodibenzo[a,c]phenazine (2DTCz-BP-F), 3,6-bis(9,9-dimethylacridin-10 $(9 H)$ yl)-10-fluorodibenzo[a,c]phenazine (2DMAC-BP-F) and 10,10'-(10fluorodibenzo[a,c]phenazine-3,6-diyl)bis(10H-phenoxazine) (2PXZBP-F). We successfully applied a modular synthesis strategy that allowed for the facile synthesis of these TADF molecules. The compounds show color tuning based on the choice of donor, emitting from green to deep-red for 2DTCz-BP-F, 2DMAC-BP-F, and 2PXZ-BP-F at peak wavelengths, $\lambda_{\mathrm{PL}}$, of $505 \mathrm{~nm}, 589 \mathrm{~nm}$, and $674 \mathrm{~nm}$ in toluene solution, respectively. 2DMAC-BP-F and 2PXZ-BP-F show small $\Delta E_{\mathrm{ST}}$ of $0.11 \mathrm{eV}$ and $0.02 \mathrm{eV}$ while using the weakest DTCz donor resulted in a compound 2DTCz-BP-F showing the largest $\Delta E_{\mathrm{ST}}$ of $0.30 \mathrm{eV}$ in doped mCBP film (5 wt $\%$ 2DTCz-BP-F, $10 \mathrm{wt} \%$ 2DMAC-BP-F, and $1.5 \mathrm{wt} \%$ 2PXZ-BP-F). The corresponding delayed lifetimes follow the trend in $\Delta E_{\mathrm{ST}}$ at $90.6 \mu \mathrm{s}, 1.83 \mu \mathrm{s}$ and $10.152 \mathrm{~ms}$, respectively, for 2DMAC-BP-F, 2PXZ-BP-F and 2DTCz-BP-F. The OLEDs fabricated with 2DMAC-BP-F, 2PXZ-BP-F and 2DTCz-BP-F exhibited EQE max $_{\text {of }}$ $21.8 \%, 12.4 \%$ and $2.1 \%$ at $\lambda_{\mathrm{EL}}$ of $585 \mathrm{~nm}, 605 \mathrm{~nm}$ and $518 \mathrm{~nm}$, respectively.

\section{Results and discussion}

\section{Molecular design and synthesis}

The synthesis of the 2D-BP-F (D = PXZ, DMAC, DTCz) family of emitters is outlined in Scheme 1. Firstly, 3,6-dibromo-10fluorodibenzo[ $[a, c]$ phenazine 3 was synthesized through a condensation reaction of 3-fluorobenzene-1,2-diamine 1 and 3,6dibromophenantrene-9,10-dione 2 in acetic acid and ethanol at elevated temperatures. Proceeding from intermediate 3 , the target compounds 2DTCz-BP-F, 2DMAC-BP-F and 2PXZ-BP-F were obtained through Pd-catalyzed Buchwald-Hartwig crosscoupling reactions in excellent yields. While the catalyst system $\mathrm{Pd}(\mathrm{OAc})_{2} / \mathrm{P}(\mathrm{Bu})_{3}$ gave excellent yields for the synthesis of 2DTCz-BP-F and 2DMAC-BP-F, the catalyst system $\mathrm{Pd}(\mathrm{dba})_{2} /$ $(t \mathrm{Bu})_{3} \mathrm{PHBF}_{4}$ gave higher yields for the synthesis of 2PXZ-BP-F.

The three emitters were purified further by gradienttemperature sublimation. The chemical structure and purity of the three compounds were confirmed using ${ }^{1} \mathrm{H},{ }^{13} \mathrm{C}$, and ${ }^{19} \mathrm{~F}$ nuclear magnetic resonance (NMR) spectroscopy, highresolution mass spectrometry (HRMS), infrared spectroscopy, melting point analysis, and elemental analysis (EA). A single crystal suitable for X-ray diffraction analysis was obtained for 2DMAC-BP-F by evaporating a solution in deuterated benzene in an NMR tube at room temperature (Fig. 2a). Analysis of the crystal structure of 2DMAC-BP-F showed that the fluorine atom is disordered about a mirror plane. The DMAC donor units are strongly twisted with a dihedral angle of $65^{\circ}$ to the BP acceptor. The DMAC donors display an almost planar conformation with the quaternary carbon being pushed out of the plane by $0.11 \AA$ while the two benzene rings of the DMAC are tilted towards each other by $4^{\circ}$. Crystals of 2DTCz-BP-F were obtained by evaporation of a solution in deuterated chloroform in an NMR tube at room temperature (Fig. 2b). Four crystallographically independent molecules were found with the fluorine atoms disordered about a mirror plane and the donor units strongly twisted with an average dihedral angle of $46.6^{\circ}$. Crystallographic data of both molecules are quoted in the ESI $\dagger$ (Table S1).

\section{Theoretical calculations}

The ground-state geometries of 2DTCz-BP-F, 2DMAC-BP-F, and 2PXZ-BP-F were optimized using density functional theory (DFT) at the PBE0/6-31G(d,p) level of theory in the gas phase. ${ }^{17,18}$ The excited state properties were calculated by time-dependent density functional theory (TD-DFT) within the Tamm-Dancoff approximation (TDA-DFT) based on the optimized ground-state geometries. ${ }^{19}$ The calculated energy levels of the highest occupied molecular orbits (HOMOs) and lowest unoccupied molecular orbits (LUMOs) are presented in Fig. 3, and the results are summarized in Table S2 (ESI $\dagger$ ). The dihedral angles between the donor and acceptor moieties were found to be around $48.6^{\circ}$ and $47.6^{\circ}$ for 2 DTCz-BP-F, $87.9^{\circ}$, and $90.1^{\circ}$ for 2DMAC-BP-F and $85.9^{\circ}$ and $72.2^{\circ}$ for 2PXZ-BP-F, respectively. In comparison with the value obtained from the crystal structure, the dihedral angle between the DMAC and the BP-F groups in 2DMAC-BP-F $\left(65^{\circ}\right)$ was found to be smaller than that 


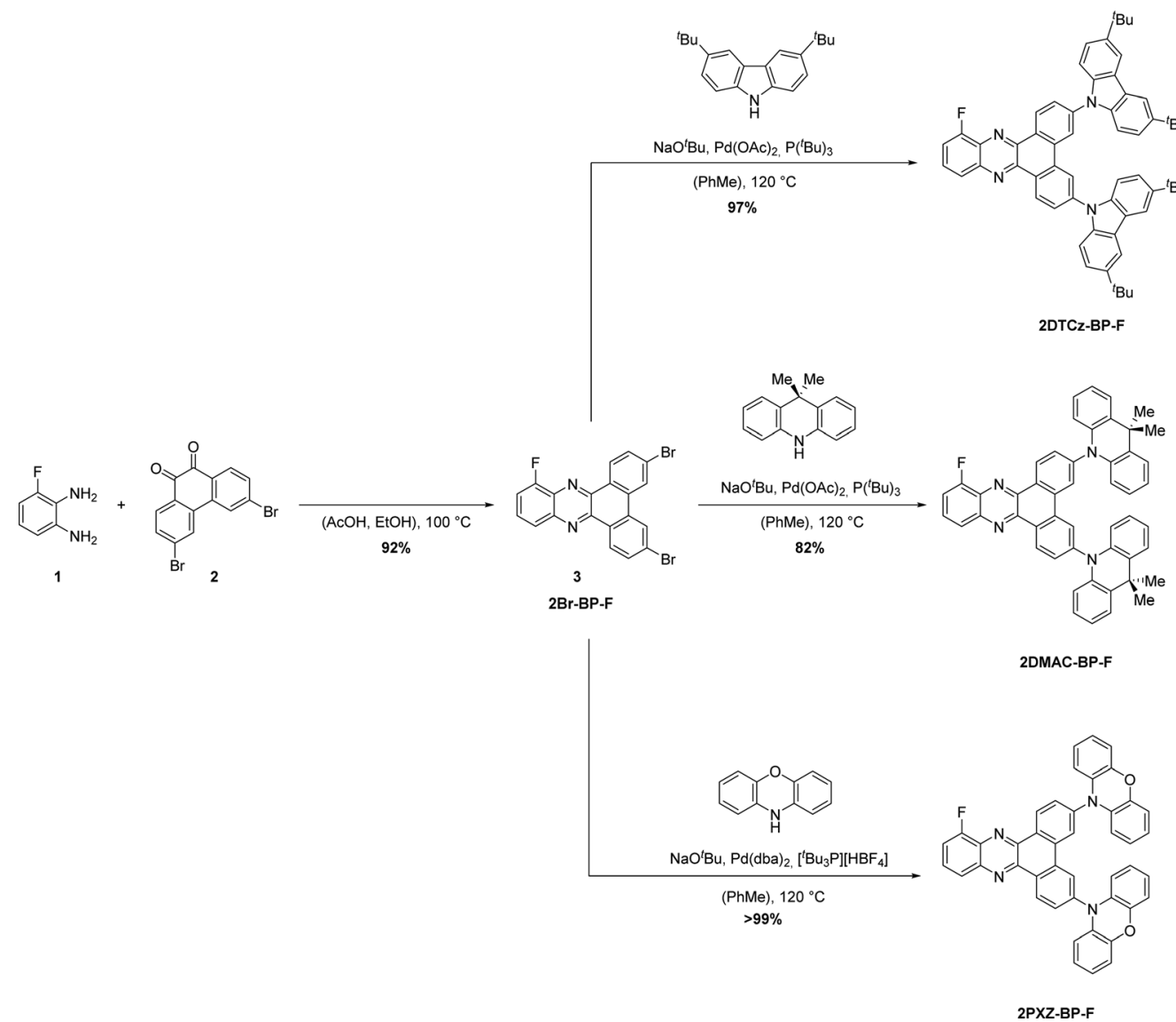

Scheme 1 Synthesis of 2DTCZ-BP-F, 2DMAC-BP-F, and 2PXZ-BP-F.

(a)



(b)

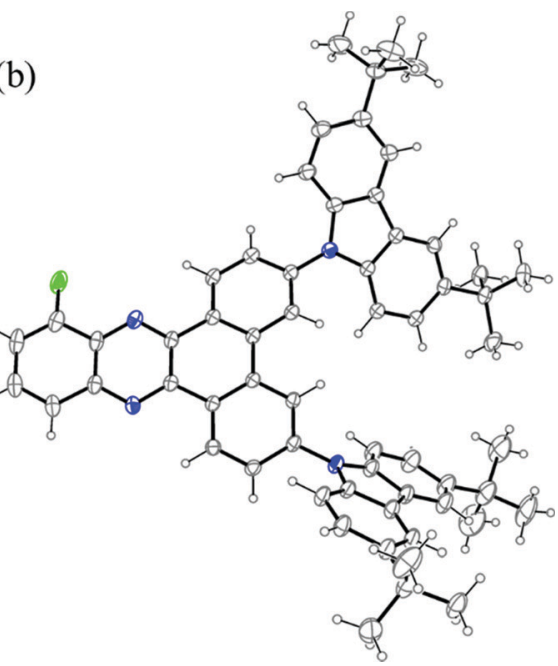

Fig. 2 (a) Molecular structure of 2DMAC-BP-F (solvent and one disordered F-position omitted for clarity, displacement parameters are drawn at 30\% probability level); (b) the molecular structure of one of the crystallographically independent molecules of 2DTCz-BP-F (solvent and minor disordered F-position omitted for clarity, displacement parameters are drawn at 30\% probability level).

theoretically calculated, while the average dihedral angle between the DTCz and the BP-F groups in 2DTCz-BP-F $\left(46.6^{\circ}\right)$ was in good accordance with the calculated value. Due to the almost orthogonal conformations of 2DMAC-BP-F and 2PXZ-BP-F, the HOMO and LUMO distributions are localized on the donor and acceptor moieties, respectively, in both molecules, which results in small $\Delta E_{\mathrm{ST}}$. The LUMOs of all three compounds are distributed over the BP-F acceptor core, while the HOMOs are 


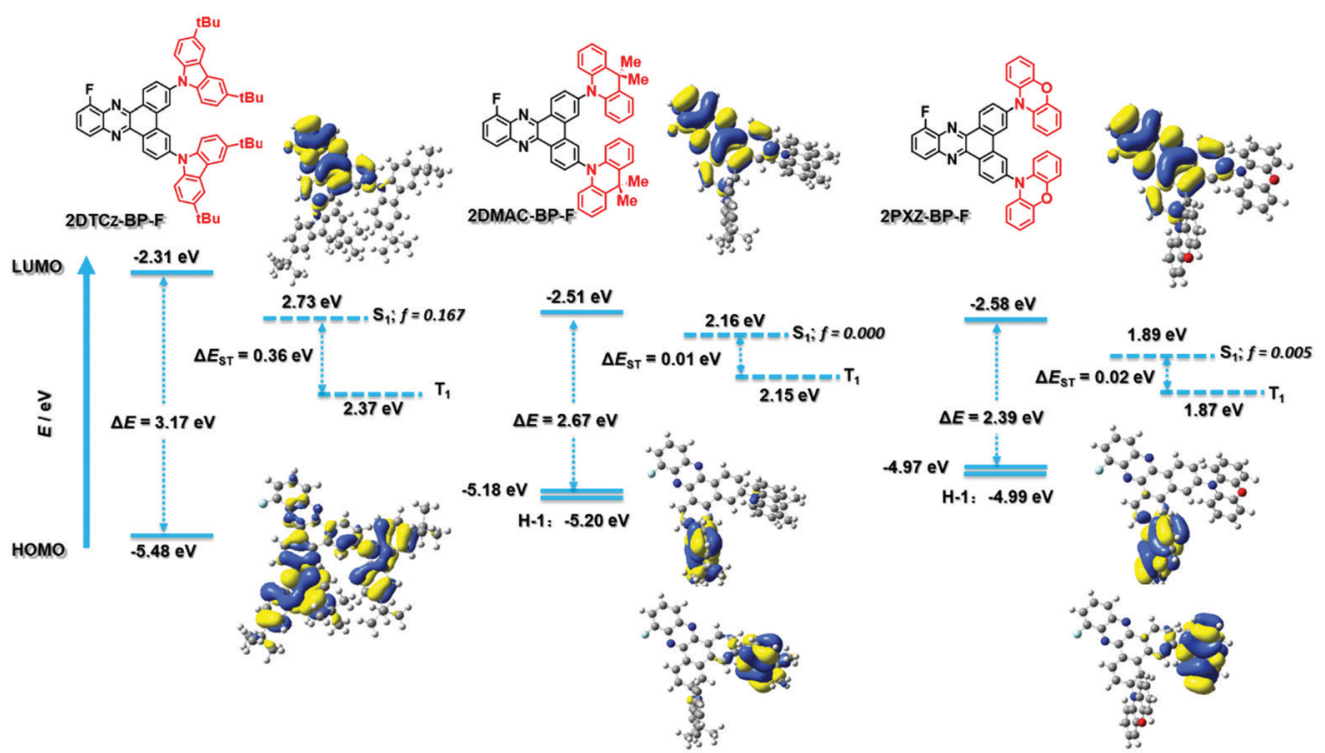

Fig. 3 Theoretical modeling of the energies of the HOMO/LUMO orbitals and the $\mathrm{S}_{1}$ and $\mathrm{T}_{1}$ states and oscillator strength of 2DTCZ-BP-F, 2DMAC-BP-F, and 2 PXZ-BP-F and the electron density distribution (ISO value $=0.02$ ) of the frontier molecular orbitals.

generally located on the donor moieties. In the case of 2DTCz-BPF, the HOMO is mainly localized on both of the 3,6-di-tert-butyl-9Hcarbazole (DTCz) units, whereas for 2DMAC-BP-F or 2PXZ-BP-F, the HOMO is mainly found on only one of the donor units while HOMO-1 is localized on the other donor as these two orbitals are pseudo degenerate (Fig. 3). The HOMO-LUMO gap, $\Delta E_{\text {Hомо-LUмO, }}$ decreases from $3.17 \mathrm{eV}$ for 2DTCz-BP-F to $2.67 \mathrm{eV}$ for 2DMAC-BP-F and $2.39 \mathrm{eV}$ for 2PXZ-BP-F, following a trend of decreasing $\Delta E_{\text {Hомо-Lumo }}$ with increasing donor strength. The oscillator strength, $f$, for the transition from $\mathrm{S}_{0}$ to $\mathrm{S}_{1}$ was found to be 0.167, 0, and 0.005 for 2DTCz-BP-Fz, 2DMAC-BP-F, and 2PXZBP-F, respectively, which reflects the significantly greater orbital overlap due to the less twisted conformation and weaker nature of the DTCz donor. The $\mathrm{S}_{1}$ energies are $2.73 \mathrm{eV}$ for 2DTCz-BP-F, $2.16 \mathrm{eV}$ for 2DMAC-BP-F, and $1.89 \mathrm{eV}$ for 2PXZ-BP-F, while the $\mathrm{T}_{1}$ energies decrease from $2.37 \mathrm{eV}, 2.15 \mathrm{eV}$, and $1.87 \mathrm{eV}$, respectively, following a similar trend to that observed for

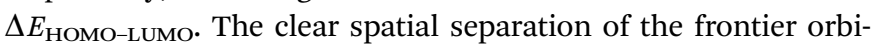
tals in 2DMAC-BP-F and 2PXZ-BP-F results in a very small $\Delta E_{\mathrm{ST}}$ of less than $0.02 \mathrm{eV}$, while the larger overlap between HOMO and LUMO for 2DTCz-BP-F leads to a $\Delta E_{\mathrm{ST}}$ of $0.36 \mathrm{eV}$.

\section{Electrochemistry}

Cyclic voltammetry (CV) was performed to determine the HOMO and LUMO levels of the emitters. The oxidation and reduction potentials of the emitters were evaluated in Arsaturated dichloromethane (DCM) solution with tetrabutylammonium hexafluorophosphate as the supporting electrolyte. The values are reported versus standard calomel electrode (SCE). The results obtained from the $\mathrm{CV}$ measurements are summarized in Table 1.

As shown in Fig. 4, all three compounds show reversible oxidation and reduction processes. The main oxidation waves occur at $0.80 \mathrm{~V}, 1.00 \mathrm{~V}$, and $1.32 \mathrm{~V}$ for 2PXZ-BP-F, 2DMAC-BP-F,
Table 1 Summary of the results obtained through cyclic voltammetry

\begin{tabular}{llllll}
\hline Emitter & $\begin{array}{l}E_{\text {ox }}{ }^{a} \\
{[\mathrm{~V} v s . \mathrm{SCE}]}\end{array}$ & $\begin{array}{l}E_{\text {red }}{ }^{a} \\
{[\mathrm{~V} v s . \mathrm{SCE}]}\end{array}$ & $\begin{array}{l}E_{\mathrm{HOMO}}{ }^{b} \\
{[\mathrm{eV}]}\end{array}$ & $\begin{array}{l}E_{\mathrm{LUMO}}{ }^{b} \\
{[\mathrm{eV}]}\end{array}$ & $\begin{array}{l}\Delta E_{\mathrm{H}-\mathrm{L}}{ }^{c} \\
{[\mathrm{eV}]}\end{array}$ \\
\hline 2PXZ-BP-F & 0.80 & -1.21 & -5.14 & -3.13 & 2.01 \\
2DMAC-BP-F & 1.00 & -1.19 & -5.34 & -3.15 & 2.20 \\
2DTCz-BP-F & 1.32 & -1.18 & -5.66 & -3.16 & 2.50
\end{tabular}

${ }^{a}$ In Ar-saturated DCM $\left(0.1 \mathrm{M}\left[n \mathrm{Bu}_{4} \mathrm{~N}\right] \mathrm{PF}_{6}\right)$ at a scan rate of $100 \mathrm{mV} \mathrm{s}^{-1}$. $E_{\text {ox/red }}[\mathrm{V} v s . \mathrm{SCE}]=E_{\text {ox/red }}\left[\mathrm{V} v s . \mathrm{Fc} / \mathrm{Fc}^{+}\right]+0.46 .{ }^{20} b$ The HOMO and LUMO energies were determined using $E_{\mathrm{HOMO} / \mathrm{LUMO}}=-\left(E_{\mathrm{ox}} / E_{\mathrm{red}}+4.8\right) \mathrm{eV}$ where $E_{\mathrm{ox}}$ and $E_{\mathrm{red}}$ are anodic and cathodic peak potentials, respectively, versus $\mathrm{Fc} / \mathrm{Fc}^{+21-23}{ }^{c} \Delta E_{\mathrm{H}-\mathrm{L}}=\left|E_{\mathrm{HOMO}}-E_{\mathrm{LUMO}}\right|$.

and 2DTCz-BP-F, respectively. These are each assigned to the oxidation of PXZ, DMAC, and DTCz, and reflect the relative strength of the donors. 2DMAC-BP-F shows an additional minor oxidation wave at $0.77 \mathrm{~V}$, which is characteristic of the redox behavior of DMAC-containing compounds. ${ }^{24}$ The respective HOMO levels are $-5.14 \mathrm{eV},-5.34 \mathrm{eV}$, and $-5.66 \mathrm{eV}$ for 2PXZ-BP-F, 2DMAC-BP-F, and 2DTCz-BP-F. The reduction waves occur at very similar potentials of $-1.21 \mathrm{~V},-1.19 \mathrm{~V}$, -1.18 V for 2PXZ-BP-F, 2DMAC-BP-F, and 2DTCz-BP-F, respectively, and indicate that the electronic coupling between the donor and acceptor moiety is small.

The corresponding redox gaps, $\Delta E_{\mathrm{H}-\mathrm{L}}$, decrease from $2.50 \mathrm{~V}$ to $2.20 \mathrm{~V}$ and $2.01 \mathrm{~V}$ for 2DTCz-BP-F, 2DMAC-BP-F, and 2PXZ-BP-F, respectively matches the HOMO-LUMO gap trend predicted by DFT calculations.

\section{Photophysical properties}

The UV-vis absorption spectra of the three emitters in dilute toluene are shown in Fig. 5a, and the photophysical properties are summarized in Table 2. All three compounds exhibit strong absorption bands at around $310 \mathrm{~nm}$, which can be attributed to locally excited (LE) $\pi-\pi^{*}$ transitions of the donors and BP-F 


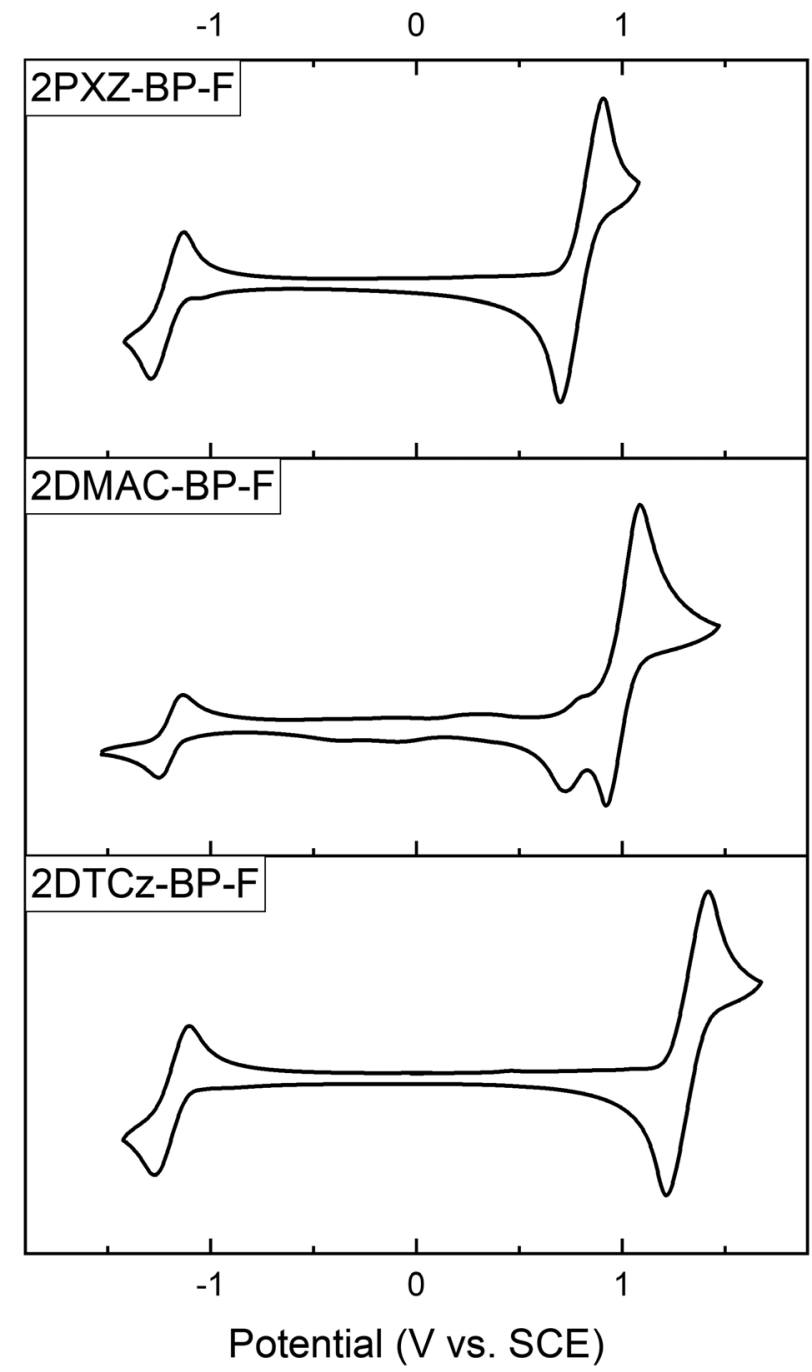

Fig. 4 Cyclic voltammograms of 2PXZ-BP-F, 2DMAC-BP-F, and 2DTCZBP-F in Ar-saturated DCM solution (0.1 $\left.\mathrm{M}\left[n \mathrm{Bu}_{4} \mathrm{~N}\right]\left[\mathrm{PF}_{6}\right]\right)$ at a scan rate of $100 \mathrm{mV} \mathrm{s}^{-1}$

moieties, respectively. ${ }^{24-26}$ Weaker and broad absorption bands are observed from 410 to $520 \mathrm{~nm}$, which are assigned to ICT transitions from the donor units to the acceptor core. ${ }^{27}$ This latter band is more intense for 2DTCz-BP-F $\left(\lambda_{\text {abs }}=440 \mathrm{~nm}, 21 \times\right.$ $\left.10^{3} \mathrm{M}^{-1} \mathrm{~cm}^{-1}\right)$ compared to those of 2DMAC-BP-F $\left(\lambda_{\mathrm{abs}}=\right.$ $\left.415 \mathrm{~nm}, 3 \times 10^{3} \mathrm{M}^{-1} \mathrm{~cm}^{-1}\right)$ and 2PXZ-BP-F $\left(\lambda_{\mathrm{abs}}=476 \mathrm{~nm}\right.$, $3 \times 10^{3} \mathrm{M}^{-1} \mathrm{~cm}^{-1}$ ) as the DTCz groups adopt a less twisted conformation, leading to greater conjugation and greater oscillator strength for the ICT transitions in 2DTCz-BP-F, values that are corroborated by the DFT calculations (Fig. S12-S17, ESI $\dagger$ ). There is the expected shift to lower energies of the ICT band across the family of compounds that is aligned with increasing donor strength. All compounds exhibit unstructured and broad PL spectra in toluene (Fig. 5a), indicative of an excited state with strong ICT character, with peak maxima, $\lambda_{\mathrm{PL}}$, at $505 \mathrm{~nm}$, $589 \mathrm{~nm}$, and $674 \mathrm{~nm}$ for 2DTCz-BP-F, 2DMAC-BP-F, and 2PXZ-BP-F, respectively. Positive solvatochromism is observed for all compounds (Fig. 5a and Table S3, ESI $\dagger$ ), which is consistent with the CT nature of the emissive excited state. The optical bandgaps, $E_{\mathrm{g}}$, calculated from the normalized absorption and emission spectra intersection point, are $2.60 \mathrm{eV}$, $2.32 \mathrm{eV}$, and $2.13 \mathrm{eV}$ for 2DTCz-BP-F, 2DMAC-BP-F, and 2PXZBP-F, respectively. Except for 2DTCz-BP-F $\left(E_{\mathrm{g}}=2.60 \mathrm{eV} v s\right.$. $\left.E_{\mathrm{S} 1 \text { theory }}=2.73 \mathrm{eV}\right)$, experimental $E_{\mathrm{g}}$ for 2DMAC-BP-F $\left(E_{\mathrm{g}}=\right.$ $\left.2.32 \mathrm{eV} v s . E_{\mathrm{S} 1 \text { theory }}=2.16 \mathrm{eV}\right)$ and 2PXZ-BP-F $\left(E_{\mathrm{g}}=2.13 \mathrm{eV} v s\right.$. $E_{\mathrm{S} 1 \text { theory }}=1.89 \mathrm{eV}$ ) were found to be larger than those calculated. The photoluminescence quantum yields, $\Phi_{\mathrm{PL}}$, in degassed toluene solution of 2DTCz-BP-F, 2DMAC-BP-F, and 2PXZ-BP-F are $51 \%, 30 \%$, and $8 \%$, respectively. These dropped to $49 \%$, $21 \%$, and $6 \%$ upon exposure to oxygen (Table 2). The prompt fluorescence and phosphorescence spectra of all compounds in 2-MeTHF at $77 \mathrm{~K}$ were measured to determine the $S_{1}$ and $T_{1}$ energies from their respective onsets (Fig. 5b and Fig. S18b, ESI, $\dagger$ Table 2). The $S_{1}$ energies of 2DTCz-BP-F, 2DMAC-BP-F, and 2PXZ-BP-F are $2.64 \mathrm{eV}, 2.47 \mathrm{eV}$, and $2.23 \mathrm{eV}$, and the $\mathrm{T}_{1}$ energies are $2.21 \mathrm{eV}, 2.27 \mathrm{eV}$, and $2.28 \mathrm{eV}$, respectively. The phosphorescence spectra of 2DTCz-BP-F and 2DMAC-BP-F are structured, and each is assigned from TDDFT calculations as a mixed locally excited triplet $\left({ }^{3} \mathrm{LE}\right)$ state of the acceptor (BP-F) and chargetransfer $\left({ }^{3} \mathrm{CT}\right)$ state (see Fig. S18a and Table S4, ESI $\dagger$ ). The phosphorescence spectrum of 2PXZ-BP-F is structureless to a mainly charge-transfer $\left({ }^{3} \mathrm{CT}\right)$ state. The $\Delta E_{\mathrm{ST}}$ of $2 \mathrm{DTCz}-\mathbf{B P}-\mathbf{F}$, 2DMAC-BP-F is $0.43 \mathrm{eV}$ and $0.20 \mathrm{eV}$, respectively. The phosphorescence spectrum of 2PXZ-BP-F in 2-MeTHF (Fig. 5 and Fig. S18b, ESI $\dagger$ ) is slightly blue-shifted compared to the prompt fluorescence, leading to an apparent $\Delta E_{\mathrm{ST}}$ of $-0.05 \mathrm{eV}$, indicating that the emission from these two states results from different conformers. To understand how the solvent polarity affects the energies of $S_{1}$ and $T_{1}$ for 2 PXZ-BP-F, we measured the prompt fluorescence and phosphorescence spectra in hexane at $77 \mathrm{~K}$, and the $\Delta E_{\mathrm{ST}}$ is $0.05 \mathrm{eV}$ for 2PXZ-BP-F (Fig. 5b bottom). We measured the PL decays of all molecules in toluene under degassed conditions using time-correlated single-photon counting (TCSPC, Fig. S19, ESI $\dagger$ ). The ICT band of 2DMAC-BP-F and 2PXZ-BP-F decays with biexponential kinetics with prompt fluorescence lifetimes, $\tau_{\mathrm{p}}$, of $27.7 \mathrm{~ns}$ and $16.9 \mathrm{~ns}$, and delayed fluorescence lifetimes, $\tau_{\mathrm{d}}$, of $19.0 \mu \mathrm{s}$ and $0.2 \mu \mathrm{s}$, respectively (Fig. S19, ESI $\dagger$ and Table 2). The delayed emission is strongly quenched upon the exposure of oxygen, indicating accessible triplet states. The ICT band of 2DTCz-BP-F decays monoexponentially with $\tau_{\mathrm{p}}$ of $6.6 \mathrm{~ns}$, no delayed emission is observed for this compound.

To assess the emission properties of these emitters in the solid-state, their photophysical properties were first investigated in PMMA. The PL spectra are unstructured with $\lambda_{\mathrm{PL}}$ at 524, 588, and $615 \mathrm{~nm}$ for 2DTCz-BP-F, 2DMAC-BP-F, and 2PXZ-BP-F, respectively (Fig. S20, ESI $\dagger$ and Table 2). The optimized $\Phi_{\mathrm{PL}}$ in doped PMMA films under an $\mathrm{N}_{2}$ atmosphere are $29 \%, 48 \%$, and $28 \%$, respectively, for 2 DTCz-BP-F $(5 \%$ in PMMA), 2DMAC-BP-F (10\% in PMMA), and 2PXZ-BP-F $(1.5 \%$ in PMMA). The $\Phi_{\mathrm{PL}}$ values were reduced to $27 \%, 34 \%$, and $17 \%$, respectively, under air. Multiexponential decay kinetics were observed in the time-resolved decays with average $\tau_{\mathrm{d}}$ values of $10.1 \mathrm{~ms}, 5.0 \mathrm{~ms}$, and $2.99 \mu$ s for 2DTCz-BP-F, 2DMAC-BP-F, and 

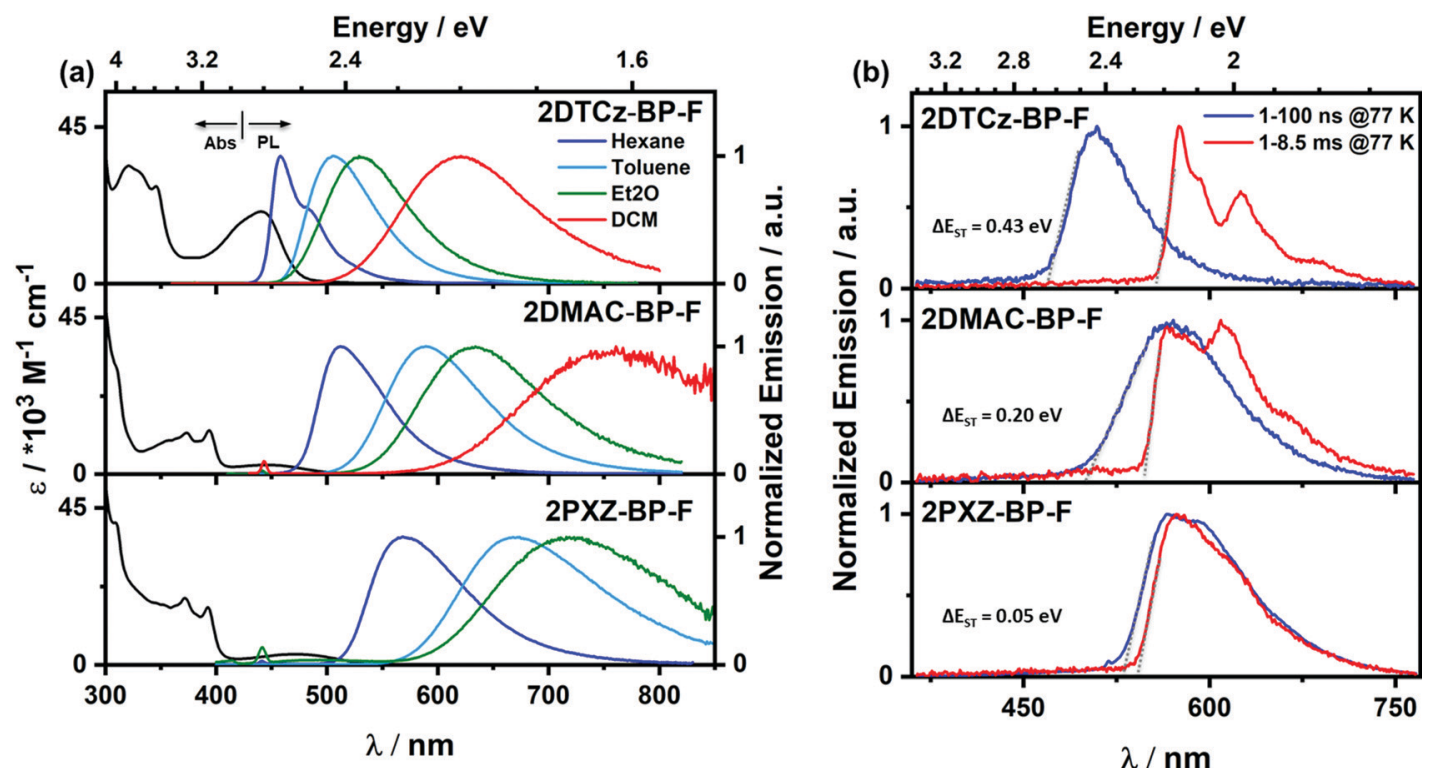

Fig. 5 (a) UV-vis absorption and PL solvatochromism study $\left(\lambda_{\text {exc }}=343 \mathrm{~nm}\right.$ ); (b) prompt fluorescence (blue line) and phosphorescence (red line) spectra of 2DTCZ-BP-F and 2DMAC-BP-F in 2-MeTHF, 2PXZ-BP-F in hexane at $77 \mathrm{~K}$ ( $\lambda_{\text {exc }}=343 \mathrm{~nm}$, prompt and delayed fluorescence spectra were obtained in the 1-100 ns and 1-8.5 ms time range, respectively). $\mathrm{Et}_{2} \mathrm{O}=$ diethyl ether; $\mathrm{DCM}=$ dichloromethane.

2PXZ-BP-F, respectively (Fig. S21, ESI $\dagger$ and Table 2). These data are consistent with TADF being operative for the emitters in PMMA films. The $\mathrm{S}_{1} / \mathrm{T}_{1}$ energy levels of 2DTCz-BP-F $(2.56 / 2.22 \mathrm{eV})$, 2DMAC-BP-F $(2.56 / 2.28 \mathrm{eV})$, and 2PXZ-BP-F $(2.34 / 2.28 \mathrm{eV})$ in PMMA films are estimated from the onsets of the fluorescence and phosphorescence spectra at $77 \mathrm{~K}$ (Fig. S22d-f, ESI $\dagger$ ). As a result, 2PXZ-BP-F $(0.06 \mathrm{eV})$ has the smallest $\Delta E_{\mathrm{ST}}$ while 2DMAC-BP-F $(0.28 \mathrm{eV})$ and 2DTCz-BP-F (0.34 eV) show significantly larger singlet-triplet gaps.

We next measured the photophysical properties of all three compounds in an OLED-relevant host 3,3'-di(9H-carbazol-9-yl)$1,1^{\prime}$-biphenyl (mCBP) as this host matrix has sufficiently high triplet energy $\left(\mathrm{T}_{1}=2.84 \mathrm{eV}\right)$ to confine the excitons onto the emitter. $^{28}$ The dopant concentration was varied from 1-10 $\mathrm{wt} \%$ in doped film to optimize the $\Phi_{\mathrm{PL}}$ (Table S5, ESI $\dagger$ ). The $\Phi_{\mathrm{PL}}$ of the $5 \mathrm{wt} \%$ 2DTCz-BP-F in mCBP doped film is $59.7 \%$ at $\lambda_{\mathrm{PL}}$ of $522 \mathrm{~nm} ; 10 \mathrm{wt} \%$ 2DMAC-BP-F in mCBP doped film is $78.0 \%$ at $\lambda_{\mathrm{PL}}$ of $584 \mathrm{~nm}$, and $1.5 \mathrm{wt} \%$ 2PXZ-BP-F in mCBP doped film is $58.0 \%$ at $\lambda_{\mathrm{PL}}$ of $611 \mathrm{~nm}$ under an $\mathrm{N}_{2}$ atmosphere (Table 2). These $\Phi_{\mathrm{PL}}$ values decreased in air to $46.8 \%$ for 2DTCz-BP-F, $48.3 \%$ for 2DMAC-BP-F, and $47.3 \%$ for 2PXZ-BP-F. All three compounds show unstructured CT-based emission in mCBP doped film at room temperature, shown in Fig. S22a-c (ESI $\dagger$ ). As shown in Fig. 6, all three compounds showed multiexponential decay kinetics with average prompt fluorescence lifetimes, average $\tau_{\mathrm{p}}$, of $4.3 \mathrm{~ns}, 19.6 \mathrm{~ns}$, and $31.0 \mathrm{~ns}$ and average delayed emission lifetimes, average $\tau_{\mathrm{d}}$, of $10.15 \mathrm{~ms}, 90.6 \mu \mathrm{s}$ and $1.83 \mu \mathrm{s}$ at room temperature for 2DTCz-BP-F, 2DMAC-BP-F, and 2PXZ-BP-F, respectively. The corresponding rate constants of intersystem crossing $\left(k_{\text {ISC }}\right)$ for the three compounds in mCBP films are $1.09 \times 10^{8} \mathrm{~s}^{-1}, 2.45 \times 10^{7} \mathrm{~s}^{-1}, 1.52 \times 10^{7} \mathrm{~s}^{-1}$ for 2DTCz-BP-F, 2DMAC-BP-F, and 2PXZ-BP-F, respectively. The rate constants of reverse intersystem crossing $\left(k_{\mathrm{RISC}}\right)$ in $\mathrm{mCBP}$ films for 2PXZ-BP-F reached $2.41 \times 10^{5} \mathrm{~s}^{-1}$, a value much faster than 2DTCz-BP-F of $5.14 \times 10^{1} \mathrm{~s}^{-1}$, and 2DMAC-BP-F of $1.33 \times$ $10^{4} \mathrm{~s}^{-1}$, respectively. The relative intensities of the delayed PL increased with increasing temperature from $100 \mathrm{~K}$ to $300 \mathrm{~K}$, thereby corroborating the TADF nature of the emission of these three compounds in the mCBP films. The extremely long lifetime and sharp decrease of the emission intensity at low temperature for the 2DTCz-BP-F doped mCBP film can be explained by the large $\Delta E_{\mathrm{ST}}$ (vide infra) and inefficient TADF.

There is an expectedly large $\Delta E_{\mathrm{ST}}$ of $0.30 \mathrm{eV}$ for 2DTCz-BP-F, while the $\Delta E_{\mathrm{ST}}$ for 2DMAC-BP-F and 2PXZ-BP-F is much smaller at $0.11 \mathrm{eV}$, and $0.02 \mathrm{eV}$, respectively. The $\mathrm{S}_{1}$ level of $2 \mathrm{DTCz}-\mathrm{BP}-\mathbf{F}$ in mCBP doped film $\left(\mathrm{S}_{1}=2.54 \mathrm{eV}\right)$ is similar to the $\mathrm{S}_{1}$ level of 2DTCz-BP-F in PMMA doped film $\left(\mathrm{S}_{1}=2.56 \mathrm{eV}\right)$ and very close to the energy level of 2DTCz-BP-F in 2-MeTHF glass $\left(\mathrm{S}_{1}=2.64 \mathrm{eV}\right)$, all of which indicates that the $S_{1}$ state in 2DTCz-BP-F is of mixed ${ }^{1} \mathrm{LE}$ and ${ }^{1} \mathrm{CT}$ character. The structured phosphorescence and triplet energy level of 2DTCz-BP-F does not change in different media such as PMMA $\left(\mathrm{T}_{1}=2.22 \mathrm{eV}\right.$, Fig. S22d, ESI $\left.\dagger\right)$, $\operatorname{mCBP}\left(\mathrm{T}_{1}=2.24 \mathrm{eV}\right.$, Fig. S22a, ESI $\left.\dagger\right)$, and in 2-MeTHF glass $\left(\mathrm{T}_{1}=2.21 \mathrm{eV}\right.$, Fig. 5b). Furthermore, these values match with the phosphorescence of the F-BP acceptor $(2.26 \mathrm{eV})$ in 2-MeTHF glass (Fig. S18a, ESI $\dagger$ ) and imply that the $\mathrm{T}_{1}$ level of $2 \mathrm{DTCz}-\mathbf{B P}-\mathbf{F}$ has ${ }^{3} \mathrm{LE}$ character. The calculated $\Delta E_{\mathrm{ST}}$ value of $2 \mathrm{DTCz}-\mathrm{BP}-\mathbf{F}$ in PMMA is $0.34 \mathrm{eV}$ and $0.30 \mathrm{eV}$ in $\mathrm{mCBP}$, values that render TADF inefficient. The weak emission band at $522 \mathrm{~nm}$ in the millisecond timescale spectra of 2DTCz-BP-F in MCBP and PMMA may be due to residual delayed fluorescence.

The $S_{1}$ levels of the other two emitters 2DMAC-BP-F and 2PXZ-BP-F in PMMA are $2.56 \mathrm{eV}$ and $2.34 \mathrm{eV}$, which are significantly blue-shifted in comparison to those in mCBP doped films, for which the $\mathrm{S}_{1}$ level for 2DMAC-BP-F is $2.40 \mathrm{eV}$, and for 2PXZ-BP-F is $2.22 \mathrm{eV}$. The $\mathrm{T}_{1}$ level of 2DMAC-BP-F is 
Table 2 Photophysical properties of 2DTCz-BP-F, 2DMAC-BP-F, and 2PXZ-BP-F

\begin{tabular}{lllllll}
\hline & $\lambda_{\mathrm{PL}}$ & $\tau_{\mathrm{p}}$ & $\tau_{\mathrm{d}}$ & $\mathrm{S}_{1} / \mathrm{T}_{1}$ & $\Delta E_{\mathrm{ST}}$ & $\Phi_{\mathrm{PL}}$ \\
Emitter & {$[\mathrm{nm}]$} & {$[\mathrm{ns}]$} & {$[\mu \mathrm{s}]$} & {$[\mathrm{eV}]$} & {$[\mathrm{eV}]$} & {$[\%]$} \\
\hline
\end{tabular}

In solution

2DTCz-BP-F

2DMAC-BP-F

2PXZ-BP-F

$\begin{array}{lll}505^{a} & 6.6^{a} & - \\ 589^{a} & 27.7^{a} & 19.0^{a} \\ 674^{a} & 16.9^{a} & 0.2^{a}\end{array}$

$2.64 / 2.21^{b}$

$2.47 / 2.27^{b}$

$0.43^{b} 51(49)^{a}$

$0.20^{b} 30(21)^{a}$

2PXZ-BP-F

$2.23 / 2.28^{b}-0.05^{b} 8(6)^{a}$

In $\mathrm{PMMA}^{c}$

$\begin{array}{lllllll}\text { 2DTCz-BP-F }(5 \mathrm{wt} \%) & 524 & - & 10105^{d} & 2.56 / 2.22 & 0.34 & 29(27)^{e} \\ \text { 2DMAC-BP-F }(10 \mathrm{wt} \%) & 588 & - & 5033^{d} & 2.56 / 2.28 & 0.28 & 48(34)^{e}\end{array}$

$\begin{array}{lllllll}\text { 2DMAC-BP-F (10 wt\%) } & 588 & - & 5033^{d} & 2.56 / 2.28 & 0.28 & 48(34)^{e} \\ \text { 2PXZ-BP-F (1.5 wt\%) } & 615 & - & 2.99^{d} & 2.34 / 2.28 & 0.06 & 28(17)^{e}\end{array}$

In $\mathrm{mCBP}^{c}$

2DTCz-BP-F (5 wt\%) $522 \quad 4.3^{d} \quad 10152^{d} \quad 2.54 / 2.24 \quad 0.30 \quad 60(47)^{e}$

$\begin{array}{lllllll}\text { 2DMAC-BP-F }(10 \mathrm{wt} \%) & 584 & 19.6^{d} & 90.6^{d} & 2.40 / 2.29 & 0.11 & 78(48)^{e}\end{array}$

$\begin{array}{lllllll}\text { 2PXZ-BP-F (1.5 wt\%) } & 611 & 31.0^{d} & 1.83^{d} & 2.22 / 2.20 & 0.02 & 58(47)^{e}\end{array}$

${ }^{a}$ At $298 \mathrm{~K}$, values quoted are in degassed toluene solutions prepared by three freeze-pump-thaw cycles: for $\lambda_{\mathrm{PL}}$ the $\lambda_{\mathrm{exc}}=343 \mathrm{~nm}$ for $2 \mathrm{DTCz}-\mathbf{B P}$ $\mathbf{F}, \lambda_{\text {exc }}=391 \mathrm{~nm}$ for 2DMAC-BP-F and 2PXZ-BP-F. For lifetime $\lambda_{\text {exc }}=$ $379 \mathrm{~nm}$. $\Phi_{\mathrm{PL}}$ values inside parenthesis quoted after aeration of a toluene solution. ${ }^{b}$ Obtained from the onset of the prompt fluorescence (time window: 1-100 ns) and phosphorescence spectra (time window: 1-8.5 ms) measured in 2-MeTHF glass at $77 \mathrm{~K}, \lambda_{\mathrm{exc}}=343 \mathrm{~nm}$. ${ }^{c}$ Thin films of PMMA and $\mathrm{mCBP}$ were prepared by spin-coating. Steady-state and time-resolved emission spectra were recorded at $298 \mathrm{~K}$ under an $\mathrm{O}_{2}$-free atmosphere $\left(\lambda_{\text {exc }}=343 \mathrm{~nm}\right.$ for steady-state and $\lambda_{\text {exc }}=379 \mathrm{~nm}$ for time-resolved emission). ${ }^{d}$ Average lifetime $\left(\tau_{\text {avg }}=\sum A_{i} \tau_{i}{ }^{2} / \sum A_{i} \tau_{i}\right.$, where $A_{i}$ is the preexponential for lifetime $\tau_{i}$ ). Prompt and delayed emissions were measured by TCSPC and MCS, respectively $\left(\lambda_{\text {exc }}=343 \mathrm{~nm}\right) .{ }^{e}$ Photoluminescence quantum yields of thin films were determined using an integrating sphere $\left(\lambda_{\text {exc }}=305 \mathrm{~nm}\right.$ or $\left.340 \mathrm{~nm}\right)$ under $\mathrm{N}_{2}$ atmosphere at $298 \mathrm{~K}$. Values quoted inside the parentheses are in the presence of $\mathrm{O}_{2}$.

2.29 eV, very similar to that in PMMA (2.28 eV for 2DMAC-BP-F) and a value that aligns with the $\mathrm{T}_{1}$ level of the BP-F acceptor in 2-MeTHF (2.26 eV, Fig. S18a, ESI $\dagger$ ). However, the $\mathrm{T}_{1}$ level of 2PXZBP-F in mCBP is $2.20 \mathrm{eV}$, which is stabilized from the value measured in PMMA at $2.28 \mathrm{eV}$ (Fig. S22f, ESI $\dagger$ and Table 2). These results reveal that the $\mathrm{T}_{1}$ state in 2DMAC-BP-F possesses dominant ${ }^{3} \mathrm{LE}$ character, while 2PXZ-BP-F shows mainly ${ }^{3} \mathrm{CT}$ character. The estimated $\Delta E_{\mathrm{ST}}$ of 2DMAC-BP-F is $0.11 \mathrm{eV}$, and of 2PXZ-BP-F is $0.02 \mathrm{eV}$ in $\mathrm{mCBP}$, while the $\Delta E_{\mathrm{ST}}$ in PMMA of 2DMAC-BP-F is $0.28 \mathrm{eV}$, and of 2PXZ-BP-F is $0.06 \mathrm{eV}$, which is suitably small for harvesting triplet excitons. Indeed, the small $\Delta E_{\mathrm{ST}}$ of 2DMAC-BP-F and 2PXZ-BP-F is a sign of an efficient TADF emitter for OLEDs.

\section{Device characterization}

OLED devices based on 2PXZ-BP-F, 2DMAC-BP-F and 2DTCzBP-F were fabricated by vacuum deposition following a typical bottom-emitting OLED device architecture (Fig. 7a) that consists of indium tin oxide (ITO)/1,4,5,8,9,11-hexaazatriphenylenehexacarbonitrile (HATCN) (5 nm) $/ N, N^{\prime}-\operatorname{di}\left(1-\right.$ naphthyl)- $N, N^{\prime}-$ diphenyl-(1,1'-biphenyl)-4, $4^{\prime}$-diamine (NPB) (40 $\left.\mathrm{nm}\right) / \operatorname{tris}(4-$ carbazoyl-9-ylphenyl)amine (TCTA) (10 nm)/emissive layer (20 nm)/1,3,5-tri[(3-pyridyl)-phen-3-yl]benzene (TmPyPB) $(40 \mathrm{~nm}) /$ $\mathrm{LiF}(0.6 \mathrm{~nm}) / \mathrm{Al}(100 \mathrm{~nm})$, where HATCN, NPB and TCTA play the role of hole injection layer (HIL), hole transportation layer (HTL) and electron blocker layer (EBL), respectively. TmPyPB acts both as electron transport layer (ETL) and hole blocking layer due to its deep HOMO $(-6.7 \mathrm{eV}),{ }^{29}$ and $\mathrm{LiF}$ acts as an electron injection layer (EIL). The molecular structures of the materials used in these OLEDs are shown in Fig. 7b. The emission layer (EML) comprises $1.5 \mathrm{wt} \%$ of 2PXZ-BP-F, $10 \mathrm{wt} \%$ 2DMAC-BP-F, or $5 \mathrm{wt} \%$ of $2 \mathrm{DTCz}-$ BP-F doped into mCBP, based on the doping study discussed above (Table S5, ESI $\dagger$ ). The performance of the OLEDs is summarized in Table 3. Current density-voltage-brightness $(J-V-L)$ curves, EQE-luminance curves, and electroluminescence spectra (EL) are given in Fig. 7c. As shown in Fig. 7e, each EL spectrum is similar to that of the corresponding PL spectrum in the thin film with EL maxima, $\lambda_{\mathrm{EL}}$, at $605 \mathrm{~nm}$ for 2PXZ-BP-F, $585 \mathrm{~nm}$ for 2DMAC-BP-F, and $518 \mathrm{~nm}$ for 2DTCz-BP-F. Similar to that observed by PL, the trend in emission energy follows that of increasing donor strength. The corresponding CIE coordinates are $(0.55,0.44),(0.51,0.48)$ and $(0.29,0.58)$ for the devices with 2PXZ-BP-F, 2DMAC-BP-F and 2DTCz-BP-F, respectively. The turn-on voltage of the devices lies between $3.3 \mathrm{~V}$ to $3.7 \mathrm{~V}$ and is dependent on the energy gap between the HOMO of materials used in HTL and EML layers. The 2DMACBP-F based device showed the best overall performance with the highest maximum external quantum efficiency $\left(\mathrm{EQE}_{\max }\right)$ of $21.8 \%$, a maximum current efficiency $\left(\mathrm{CE}_{\max }\right)$ of $59.7 \mathrm{~cd} \mathrm{~A}^{-1}$, and maximum power efficiency $\left(\mathrm{PE}_{\mathrm{max}}\right.$ ) of $55.4 \mathrm{~lm} \mathrm{~W}^{-1}$ (Table 3, Fig. 7d and Fig. S23 and S24, ESI $\dagger$ ). The $\mathrm{EQE}_{\max }$ of the 2PXZ-BP-F-based device is $12.4 \%$ with $\mathrm{CE}_{\max }=26.3 \mathrm{~cd} \mathrm{~A}^{-1}$ and $\mathrm{PE}_{\max }=23.0 \mathrm{~lm} \mathrm{~W}^{-1}$. The 2PXZ-BP-F-based device showed moderate roll-off efficiency, with the EQE at $100 \mathrm{~cd} \mathrm{~m}^{-2}$ at $9.3 \%$ and the EQE at $1000 \mathrm{~cd} \mathrm{~m}^{-2}$ at 6.3\%. The 2DMAC-BP-F-based device, however, showed higher efficiency roll-off, with an EQE at $100 \mathrm{~cd} \mathrm{~m}^{-2}$ of $8.7 \%$ and an
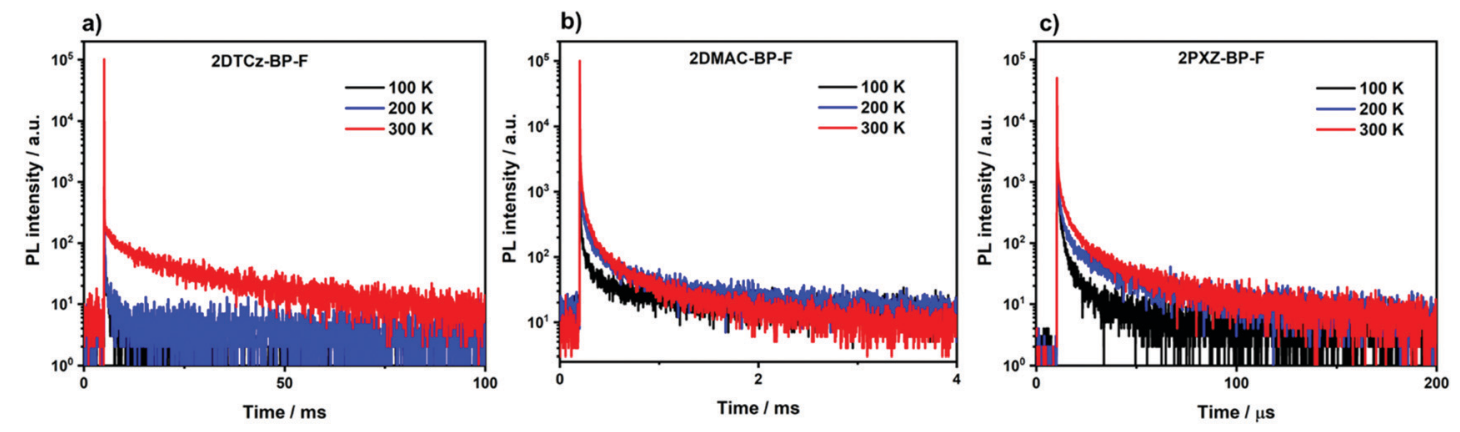

Fig. 6 Temperature-dependent time-resolved PL decay of (a) 5 wt\% 2DTCz-BP-F doped mCBP film; (b) 10 wt\% 2DMAC-BP-F doped mCBP film; (c) 1.5 wt\% 2PXZ-BP-F doped mCBP film ( $\lambda_{\text {exc }}=379 \mathrm{~nm}$ ). 

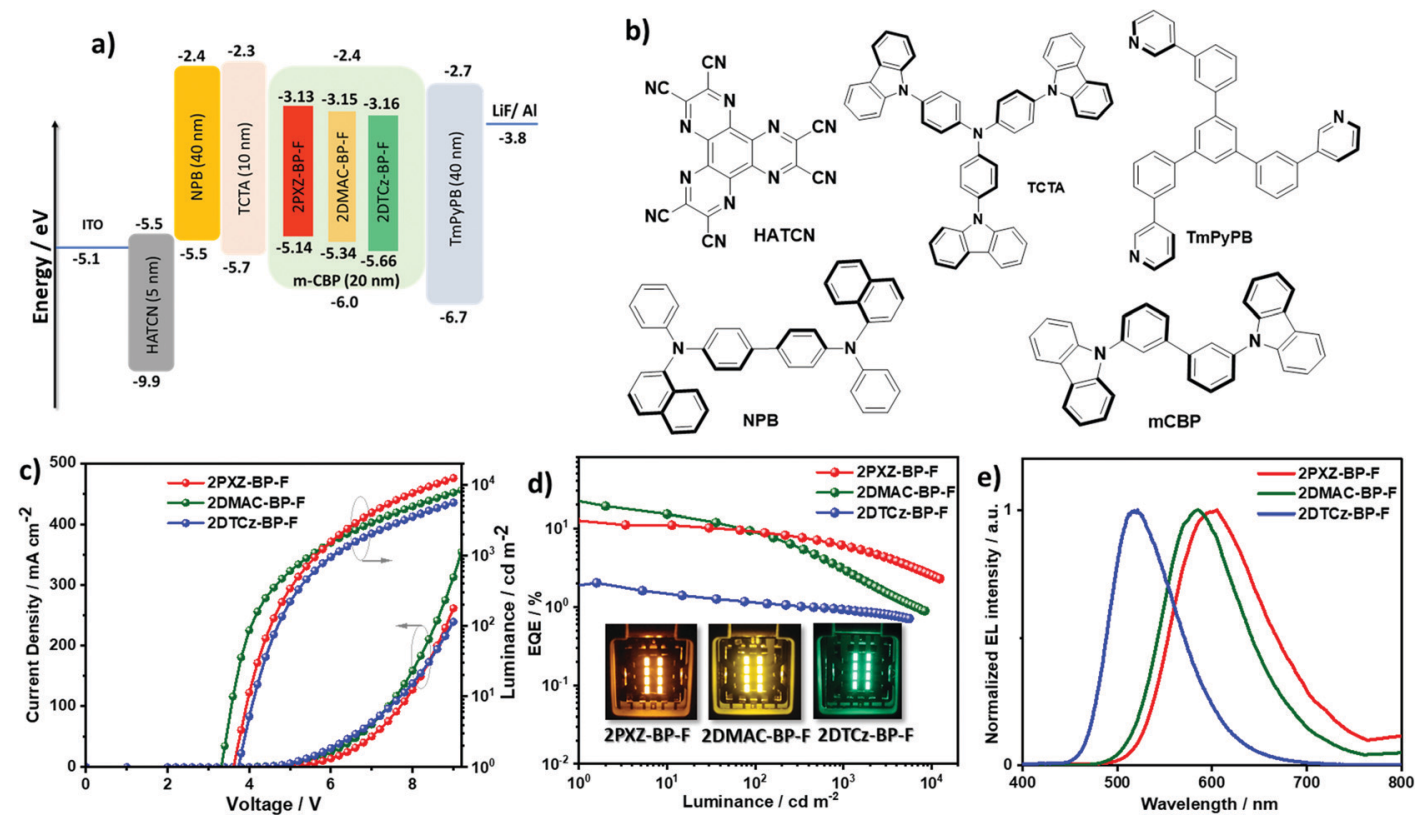

Fig. 7 (a) Energy level diagram of materials employed in the devices; (b) molecular structure of materials used in the devices; (c) current density and luminance versus voltage characteristics for the devices; (d) external quantum efficiency versus luminance curves for the devices, the inset is the electroluminescence of 2PXZ-BP-F, 2DMAC-BP-F, and 2DTCZ-BP-F; (e) electroluminescence spectra of the device.

Table 3 Electroluminescence data for the devices ${ }^{a}$

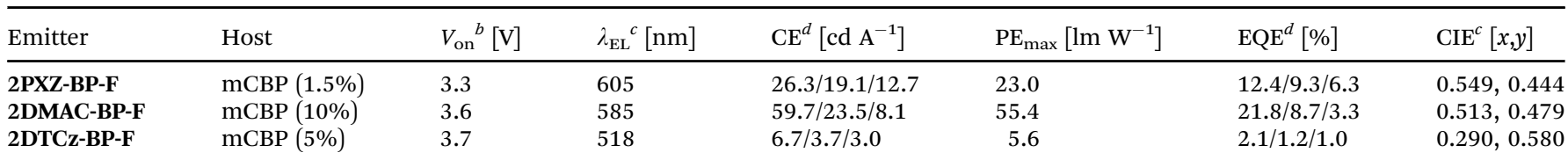

${ }^{a}$ Device stacks; ITO/HATCN $(5 \mathrm{~nm}) / \mathrm{NPB}(40 \mathrm{~nm}) / \mathrm{TCTA}(10 \mathrm{~nm}) / \mathrm{emissive}$ layer $(20 \mathrm{~nm}) / \mathrm{TmPyPB}(40 \mathrm{~nm}) / \mathrm{LiF}(0.6 \mathrm{~nm}) / \mathrm{Al}(100 \mathrm{~nm}) .{ }^{b}$ The turn-on voltage at a brightness $1 \mathrm{~cd} \mathrm{~m}^{-2} .{ }^{c}$ The electroluminescence maximum and CIE coordinates recorded at $5 \mathrm{~V}$. ${ }^{d}$ The order of measured values: the maximum EQE/EQE at $100 \mathrm{~cd} \mathrm{~m}^{-2} / \mathrm{EQE}$ at $1000 \mathrm{~cd} \mathrm{~m}^{-2}$.

EQE of $1000 \mathrm{~cd} \mathrm{~m}^{-2}$ at $3.3 \%$. The maximum brightness of the 2PXZ-BP-F-based device reached $12350 \mathrm{~cd} \mathrm{~m}^{-2}$ at an EQE of $2.3 \%$. The relatively low efficiency roll-off in 2PXZ-BP-F originates in part from the low triplet exciton concentration due to the relatively short delayed lifetime $\left(\tau_{\mathrm{d}}=1.83 \mu \mathrm{s}\right) .{ }^{30,31}$ Notably, the 2PXZ-BP-Fbased device reached an EQE of $2.5 \%$ at $10000 \mathrm{~cd} \mathrm{~m}^{-2}$ with an emission wavelength beyond $600 \mathrm{~nm}$. Although 2DTCz-BP-F shows a high $\Phi_{\mathrm{PL}}$ of $c a .60 \%$ in the $5 \mathrm{wt} \%$ doped in $\mathrm{mCBP}$, the device exhibits a low $\mathrm{EQE}_{\max }$ of $2.1 \%$. As a result of the too high $\Delta E_{\mathrm{ST}}$, the harvesting of triplet excitons in the 2DTCz-BP-F-based device is very inefficient as reflected in the very long delayed lifetime, which causes more triplet-triplet annihilation and triplet-polaron annihilation. Devices fabricated using $\mathrm{MoO}_{3}$ as the HIL showed similar performance but reached lower luminance and low current density than the devices using HATCN (Table S6 and Fig. S25, ESI $\dagger$ ).

\section{Conclusions}

This study reported a series of green-to-red-emitting fluorine-substituted dibenzo[a,c]phenazine-based (BP-F) TADF emitters. 2DTCzBP-F, 2DMAC-BP-F, and 2PXZ-BP-F, which showed color tuning based on the choice of donor, emitting from green to deep-red.
The rigid and planar constituent groups with large steric hindrance between donor and acceptor units endow these emitters with high $\Phi_{\mathrm{PL}}$ vlues and suitably small $\Delta E_{\mathrm{ST}}$. Among them, 2DMAC-BP-F exhibits the highest $\Phi_{\mathrm{PL}}$, at $78 \%$, a relatively small $\Delta E_{\mathrm{ST}}$ of $0.11 \mathrm{eV}$ at $584 \mathrm{~nm}$ in $10 \mathrm{wt} \%$ doped mCBP, whereas 2PXZ-BP-F shows the smallest $\Delta E_{\mathrm{ST}}$ of $0.02 \mathrm{eV}$ with shortest delay lifetime of $1.83 \mu \mathrm{s}$ at $611 \mathrm{~nm}$ in $1.5 \mathrm{wt} \%$ doped mCBP. OLED devices using these TADF materials showed excellent performance with an $\mathrm{EQE}_{\max }$ of $21.8 \%$ in the case of 2DMAC-BP-F with $\lambda_{\mathrm{EL}}$ of $585 \mathrm{~nm}$ and $12.4 \%$ for 2 PXZBP-F with $\lambda_{\mathrm{EL}}$ of $605 \mathrm{~nm}$. The relatively low efficiency roll-off in 2PXZBP-F is due to the short delayed lifetime, making this material a very good TADF emitter for OLEDs in the family of devices that can reach brightness above $10000 \mathrm{~cd} \mathrm{~m}^{-2}$ with an emission wavelength beyond $600 \mathrm{~nm}$. These results demonstrate that simple modification of the BP acceptor with a fluorine substituent is an effective approach to design orange-red/red TADF emitters with devices that show high EQE and low-efficiency roll-off.

\section{Data availability}

The research data supporting this publication can be accessed at https://doi.org/10.17630/2553f890-29d1-4fb8-a49c-0cf06404d856. 


\section{Conflicts of interest}

The authors declare no conflict of interest.

\section{Acknowledgements}

The authors would like to thank the RTG 2039 "Molecular Architectures for Fluorescent Cell Imaging” by the Deutsche Forschungsgemeinschaft (DFG) for financial support. C. S. thanks the China Scholarship Council (201806890001). We thank Dr Tomas Matulaitis for help with $\Delta E_{\mathrm{ST}}$ measurements. A. K. G. is grateful to the Royal Society for Newton International Fellowship NF171163. We acknowledge support from the UK's Engineering and Physical Sciences Research Council (grants EP/P010482/1 and EP/L017008/1). E. Z.-C. is a Royal Society Leverhulme Trust Senior Research fellow (SRF/R1/201089).

\section{References}

1 M. Y. Wong and E. Zysman-Colman, Adv. Mater., 2017, 29, 1605444.

2 G. Hong, X. Gan, C. Leonhardt, Z. Zhang, J. Seibert, J. M. Busch and S. Brase, Adv. Mater., 2021, 33, e2005630.

3 P. de Silva, C. A. Kim, T. Zhu and T. Van Voorhis, Chem. Mater., 2019, 31, 6995-7006.

4 J. X. Chen, W. W. Tao, W. C. Chen, Y. F. Xiao, K. Wang, C. Cao, J. Yu, S. Li, F. X. Geng, C. Adachi, C. S. Lee and X. H. Zhang, Angew. Chem., Int. Ed., 2019, 58, 14660-14665.

5 J. Xue, Q. Liang, R. Wang, J. Hou, W. Li, Q. Peng, Z. Shuai and J. Qiao, Adv. Mater., 2019, 31, e1808242.

6 R. Englman and J. Jortner, Mol. Phys., 1970, 18, 145-164.

7 J. V. Caspar, E. M. Kober, B. P. Sullivan and T. J. Meyer, J. Am. Chem. Soc., 2002, 104, 630-632.

8 J. Eng and T. J. Penfold, Chem. Rec., 2020, 20, 831-856.

9 J. H. Kim, J. H. Yun and J. Y. Lee, Adv. Opt. Mater., 2018, 6, 1800255.

10 Q. Zhang, H. Kuwabara, W. J. Potscavage, Jr., S. Huang, Y. Hatae, T. Shibata and C. Adachi, J. Am. Chem. Soc., 2014, 136, 18070-18081.

11 W. Zeng, H. Y. Lai, W. K. Lee, M. Jiao, Y. J. Shiu, C. Zhong, S. Gong, T. Zhou, G. Xie, M. Sarma, K. T. Wong, C. C. Wu and C. Yang, Adv. Mater., 2018, 30, 1704961.

12 L. Yu, Z. Wu, G. Xie, W. Zeng, D. Ma and C. Yang, Chem. Sci., 2018, 9, 1385-1391.
13 F. M. Xie, P. Wu, S. J. Zou, Y. Q. Li, T. Cheng, M. Xie, J. X. Tang and X. Zhao, Adv. Electron. Mater., 2020, 6, 1900843.

14 F. M. Xie, H. Z. Li, G. L. Dai, Y. Q. Li, T. Cheng, M. Xie, J. X. Tang and X. Zhao, ACS Appl. Mater. Interfaces, 2019, 11, 26144-26151.

15 S. Kothavale, W. J. Chung and J. Y. Lee, ACS Appl. Mater. Interfaces, 2020, 12, 18730-18738.

16 Y. Liu, Y. Chen, H. Li, S. Wang, X. Wu, H. Tong and L. Wang, ACS Appl. Mater. Interfaces, 2020, 12, 30652-30658.

17 C. Adamo and V. Barone, J. Chem. Phys., 1999, 110, 6158-6170.

18 S. Grimme, Chem. Phys. Lett., 1996, 259, 128-137.

19 S. Hirata and M. Head-Gordon, Chem. Phys. Lett., 1999, 314, 291-299.

20 N. G. Connelly and W. E. Geiger, Chem. Rev., 1996, 96, 877-910.

21 A. J. Bard and L. R. Faulkner, Electrochemical Methods: Fundamentals and Applications, John Wiley \& Sons, 1980.

22 M. Thelakkat and H.-W. Schmidt, Adv. Mater., 1998, 10, 219-223.

23 J. L. Brédas, R. Silbey, D. S. Boudreaux and R. R. Chance, J. Am. Chem. Soc., 2002, 105, 6555-6559.

24 S. Kothavale, W. J. Chung and J. Y. Lee, J. Mater. Chem. C, 2020, 8, 7059-7066.

25 J. X. Chen, K. Wang, C. J. Zheng, M. Zhang, Y. Z. Shi, S. L. Tao, H. Lin, W. Liu, W. W. Tao, X. M. Ou and X. H. Zhang, Adv. Sci., 2018, 5, 1800436.

26 Y.-Y. Wang, Y.-L. Zhang, K. Tong, L. Ding, J. Fan and L.-S. Liao, J. Mater. Chem. C, 2019, 7, 15301-15307.

27 J. X. Chen, W. W. Tao, Y. F. Xiao, K. Wang, M. Zhang, X. C. Fan, W. C. Chen, J. Yu, S. Li, F. X. Geng, X. H. Zhang and C. S. Lee, ACS Appl. Mater. Interfaces, 2019, 11, 29086-29093.

28 S. Gong, X. He, Y. Chen, Z. Jiang, C. Zhong, D. Ma, J. Qin and C. Yang, J. Mater. Chem., 2012, 22, 2894-2899.

29 S. J. Su, T. Chiba, T. Takeda and J. Kido, Adv. Mater., 2008, 20, 2125.

30 A. K. Gupta, W. Li, A. Ruseckas, C. Lian, C. L. CarpenterWarren, D. B. Cordes, A. M. Z. Slawin, D. Jacquemin, I. D. W. Samuel and E. Zysman-Colman, ACS Appl. Mater. Interfaces, 2021, 13, 15459-15474.

31 N. C. Giebink and S. R. Forrest, Phys. Rev. B: Condens. Matter Mater. Phys., 2008, 77, 235215. 